\title{
Effect of Fin Height and Rayleigh Number with Small Increments on Convective Heat Transfer in a Horizontal Annulus
}

\author{
Anas El Amraoui, Abdelkhalek Cheddadi*, Mohammed T. Ouazzani \\ MOSEM2PI Laboratory, Mohammed V University in Rabat, Mohammadia School of Engineers, Ibn Sina Str., Rabat 10090 , \\ Morocco
}

Corresponding Author Email: cheddadi@emi.ac.ma

https://doi.org/10.18280/ijht.380207

Received: 4 November 2019

Accepted: 20 May 2020

\section{Keywords:}

natural convection, fins, heat transfer, effectiveness, horizontal annulus

\begin{abstract}
The purpose of this paper is to investigate numerically the laminar natural convection in an annular space bounded by two coaxial horizontal cylinders equipped with two fins of height $h$, ranging from 0.015 to 0.953 . Rayleigh number, $R a$, is considered between $10^{3}$ and $10^{4}$. The effect of the fin height and Rayleigh number, both varied with small increments is analyzed. The resulting flow structures and heat transfer rates as well as the effectiveness of the system are presented. The results show that for each $R a$ the increase in $h$ leads to a decrease or an increase in heat transfer rate depending on the fins height value, and that the case without fins can be more favorable to the heat transfer rate compared to the case with fins for intervals of $h$ obtained as a function of $R a$. It is also shown that the increase in the Rayleigh number does not necessarily correspond to an increase in heat transfer rate. In particular, the disappearance of the bicellular regime leads to a decrease of the heat transfer rate when $R a$ increases.
\end{abstract}

\section{INTRODUCTION}

The study of natural convection in differentially heated and finned cavities designed to control heat transfer rate has been of great interest in recent decades and remains important in thermal sciences research due to the involvement of this device in many applications such as cooling of electronic components [1], heat exchangers and annular nuclear reactor, etc. In many applications such as building insulation and storage, the goal is to reduce heat transfer rate, while in others, such as the heat sink the increase in heat transfer rate is the objective sought, since natural convection cooling remains noiseless and at low cost.

Some of the published research has focused on analyzing the heat transfer rate by acting on the height of the fins. Chai and Patankar [2] studied the effect of the orientation and the height of the fins on the natural convection between two horizontal cylinders with internal fins of height ranging from 0.2 to 0.7 , and Rayleigh number values equal to $10^{3}, 10^{4}, 10^{5}$ and $10^{6}$. The results show that the internal orientation of the fins has a negligible effect on the average Nusselt number ratio (ratio of heat transfer rate to that of pure conduction) which increases with the increase of the Rayleigh number and decreases with the increase of the height of the fins. The authors found that long fins block and weaken the fluid flow. Similarly, it has been shown by Rahnama et al. [3] that using fins enhances the heat transfer rate and that the effect of their orientation is negligible. On the other hand, the long fins block the circulation of the flow reducing the average Nusselt number ratio. Afterwards, the numerical study conducted by Rahnama et al. [4] on turbulent natural convection in the presence of radial fins of height equal to $0.2,0.4$ and 0.8 with a number of fins that ranges from 2 up to 12 for Rayleigh numbers ranging from $10^{6}$ to $10^{9}$, showed that the effectiveness is less than 1 for all configurations. It decreases further with the increase of the height of the fins, reducing the rate of heat transfer compared to the case without fins. They also showed that the Nusselt number increases with the increase in the Rayleigh number. The results obtained by Jafari et al. [5] indicate that in a medium limited by a cylinder and a square, the more Rayleigh number increases the more Nusselt number also increases. Mixed convection is analyzed by Soliman and coworkers $[6,7]$ in horizontal tubes with radial internal fins. The results indicate that the fins suppress the free convection currents and therefore, the average Nusselt number ratio improvement decreases as the height of the fins increases. Abu-Hijleh et al. [8] studied the effect of several combinations of number and height of low conductivity fins on the normalized Nusselt number (average Nusselt number with baffles to average Nusselt number at cylinder surface) for a wide range of Rayleigh number. The results showed that there is an optimal combination of the number of fins and their heights that minimizes the normalized Nusselt number.

Farinas and coworkers $[9,10]$ studied the effect of fins with height equal to $0.25,0.3,0.5$ and 0.75 placed inside a horizontal annular cavity and a horizontal rhombic cavity, on the fluid flow, the distribution of temperature and on the average Nusselt number for Rayleigh numbers ranging from $10^{3}$ to $10^{6}$. The longest fin has been shown to generate a flow with a smaller stagnant area and a more convective flow that contributes more efficiently to heat transfer rate. They also found that the fin effectiveness decreases as Rayleigh number increases. Alshahrani and Zeitoun [11] also demonstrated that increasing the height of the fins for different inclinations increases the total heat transfer which also increases with increasing Rayleigh number. The use of unequal heights fins in annular cavities is adopted by Zeitoun and Hegazy [12] to improve Nusselt number. Elatar et al. [13] have shown that 
increasing fin height and conductivity ratio causes increased fin effectiveness in a vertical-walled square cavity. The numerical study of the laminar natural convection of nanofluids $[14,15]$ between two radial concentric cylinders with radial fins fixed to the inner cylinder has shown that as the height and the number of fins increase, the fin effectiveness increases with a greater effect of the height. As the Rayleigh number increases the buoyancy force is intensified and thus the Nusselt number is improved. The use of two porous fins attached to the inner cylinder has shown that increasing the Rayleigh number increases the average Nusselt number in an annular cavity as studied by Kiwan and Zeitoun [16]. Masliyah and Nandakumar [17] found that Nusselt number increases with increasing fin height in a circular tube with internal fins. The optimization of the finned pipe configuration as a function of fin height, number of fins, fin thickness, and ratio of inner and outer tube radii is investigated by Iqbal and coworkers [18-20] to improve the heat transfer rate coefficient. Various optimal configurations have been proposed based on practical and industrial requirements. The study of the effect of changing initial conditions carried out by Idrissi et al. [21], showed the existence of a bifurcation point separating two flow regimes: uni- and bi-cellular. The bicellular regime leads to substantial enhancement of the heat transfer rate compared to the unicellular one.

Most of the studies presented previously, in the analysis of the phenomenon of convection with fins, take into account the effect of several geometrical and thermo-physical parameters that can improve the heat transfer. Despite the large number of scientific contributions related to thermal analysis in finned cylindrical cavities, the variation of the height of the fins and Rayleigh number remains among the most common and practical ways whose effect on heat transfer is important and continues to be studied and analyzed. The present paper focuses for the first time to the best of our knowledge, on the study of the effect of fin height considered with a very tight increment, on heat transfer rate and fluid flow. The effect of Rayleigh number varied with small increment is also analyzed to determine the conditions required for increasing or decreasing the heat transfer rate.

\section{MATHEMATICAL MODELING OF THE PROBLEM}

The schematic of the geometry analyzed in this study is shown in Figure 1, consisting of an annular space filled with air $(\operatorname{Pr}=0.7)$, bounded by two isothermal cylinders, coaxial, horizontal, very long, which allows us to consider the problem as two-dimensional. The cylinder walls and the two fins are assumed to be highly conducting, in order that temperatures remain uniform along the cylindrical walls and the fins. The isothermal fins are arranged on the hot wall of temperature $T_{i}$ (inner cylinder of radius $r_{i}$ ) symmetrically with respect to the vertical plane containing the axis of the cylinders. The cold wall (outer cylinder of radius $\left.r_{o}\right)$ is at a temperature $T_{o}\left(T_{o}<\right.$ $\left.T_{i}\right)$. The radius ratio of the cylinders is $R=r_{o} / r_{i}=2$. The fins are placed at the angular position $\varphi_{m}=0.82 \pi$ from downward and have a dimensionless height $h$ defined with respect to $r_{i}$ and a very low dimensionless thickness of value 0.015 defined with respect to the inner half-perimeter $\left(\pi r_{i}\right)$. The choice of this fins position is justified by the fact that in the finless case, the bicellular regime which appears at the top of the annulus [22] increases the heat transfer rate, and that in the case with fins with the height 0.140 and the thickness 0.109 , it has been shown that this position rises the heat transfer rate relatively to others [23].

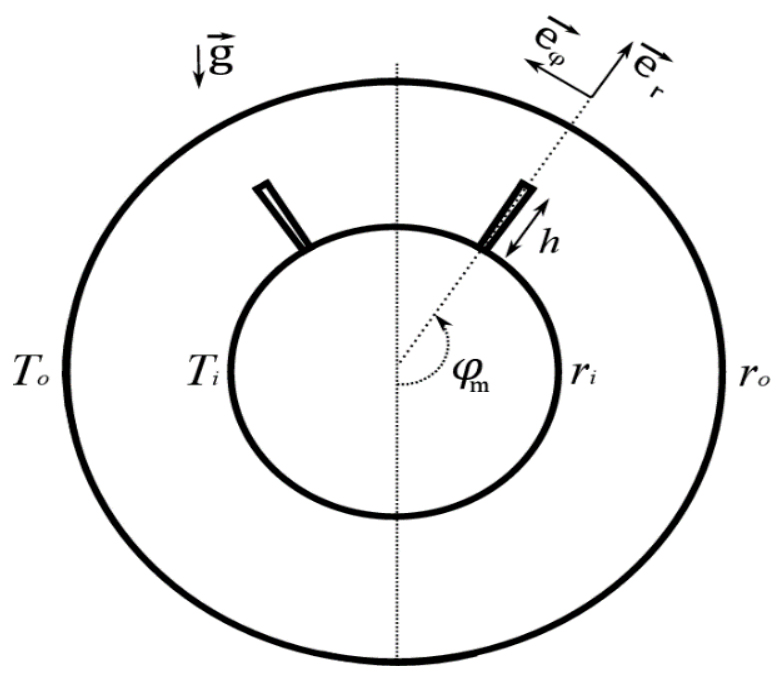

Figure 1. Schematic of the geometry

The fluid occupying the annular space is considered to be incompressible and viscous, obeying the Boussinesq approximation. The problem considered is laminar and steady. The governing conservation equations, written in nondimensional form using the vorticity-stream function formulation $(\omega, \psi)$, are given by:

$$
\Delta \psi+\omega=0
$$

$$
\begin{gathered}
\frac{\partial \omega}{\partial t}+U \frac{\partial \omega}{\partial r}+\frac{V}{r} \frac{\partial \omega}{\partial \varphi}=\operatorname{Pr} \Delta \omega+\operatorname{RaPr}\left(\sin \varphi \frac{\partial T}{\partial r}+\frac{\cos \varphi}{r} \frac{\partial T}{\partial \varphi}\right) \\
\frac{\partial T}{\partial t}+U \frac{\partial T}{\partial r}+\frac{V}{r} \frac{\partial T}{\partial \varphi}=\Delta T
\end{gathered}
$$

The stream function is related to the radial and tangential components of the dimensionless velocity by the following relations:

$$
U=\frac{1}{r} \frac{\partial \psi}{\partial \varphi}, V=-\frac{\partial \psi}{\partial r}
$$

$R a$ and $P r$ are respectively the Rayleigh and Prandtl numbers defined by:

$$
R a=\frac{g \beta\left(T_{i}-T_{o}\right) r_{i}^{3}}{v \alpha}, \operatorname{Pr}=\frac{v}{\alpha}
$$

The physical quantities $\alpha, v$ and $\beta$ are respectively: the thermal diffusivity, the kinematic viscosity and the coefficient of thermal expansion.

The boundary conditions write:

- On the inner wall:

$$
r=1: \psi=0, \frac{\partial \psi}{\partial r}=0, \frac{\partial^{2} \psi}{\partial r^{2}}+\omega=0, T=1
$$

- On the outer wall: 


$$
r=R: \psi=0, \frac{\partial \psi}{\partial r}=0, \frac{\partial^{2} \psi}{\partial r^{2}}+\omega=0, T=0
$$

- On the border of the isothermal fins:

$$
\begin{aligned}
& \psi=0, \frac{\partial \psi}{\partial \varphi}=0, \frac{1}{r^{2}} \frac{\partial^{2} \psi}{\partial \varphi^{2}}+\omega=0, T=1 \text { for } \\
& 1 \leq r \leq 1+h \\
& \psi=0, \frac{\partial \psi}{\partial r}=0, \frac{\partial^{2} \psi}{\partial r^{2}}+\omega=0, T=1 \text { at } r=1+h
\end{aligned}
$$

It is furthermore considered that the problem is symmetrical. The symmetry conditions write:

$$
\varphi=0 \quad \text { and } \quad \varphi=\pi: \psi=0, \omega=0, \quad \frac{\partial T}{\partial \varphi}=0 \quad \forall r
$$

The numerical model used to solve the governing Eqns. (13) iteratively is based on the Centered Finite Difference method with the ADI (Alternating Direction Implicit) scheme. Thomas algorithm is applied to solve the obtained tri-diagonal matrix systems. The initialization of the calculations is processed by the introduction of zero fields of the vorticity and stream function, and a pure conduction temperature field. The results are obtained for steady-state flows. In order to keep the convergence time to a minimum without losing accuracy a $65 \times 65$ grid was found sufficient. The validation was carried out by comparing the results of the heat transfer rate obtained at $h=0$ with those obtained in the case without fins studied in a previous work by Cheddadi et al. [22]. The results show a good agreement since the difference that does not exceed $0.24 \%$ found for $R a=10^{4}$.

The local Nusselt number along the outer cylinder is calculated as the ratio of convective to conductive heat transfer:

$$
N u_{o}(r=R, \varphi)=-\left.R \ln R \frac{\partial T}{\partial r}\right|_{r=R}
$$

The average heat transfer rate defined by the Nusselt at the cold wall is given by:

$$
\overline{N u}=\frac{1}{\pi} \int_{0}^{\pi} N u_{o} d \varphi=-\left.\frac{1}{\pi} R \ln R \int_{0}^{\pi} \frac{\partial T}{\partial r}\right|_{r=R} d \varphi
$$

\section{RESULTS AND DISCUSSION}

The present work investigates the effect of the fin height $h$, and of Rayleigh number $R a$, on the flow structure and the heat transfer rate through the cylindrical annular cavity. The fin height is ranging from 0.015 to 0.953 with the increment of 0.015 and the Rayleigh number varies from $10^{3}$ to $10^{4}$ incremented by 10 units in some cases where the need arises.

During the variation of $h$ for the Rayleigh numbers studied, different flow regimes are observed. At low heights, the unicellular regime, UCR, established is similar to that of the finless case. The increase in $h$ shows for the low $R a(1000 \leq$ $R a \leq 2790$ ) the appearance of a small cell near to the fin, indicating that the regime is bicellular, BCR. With the continuous increase in height different regimes appear: bicellular-bi-vortex, BCBV, characterized by the presence of a main cell with two vortices of different sizes in addition to a secondary cell, bicellular-tri-vortex, BCTV, quad-cellular, QCR, where four cells are noticed, then tricellular, TCR. Figure 2 (a) illustrates these regimes for $R a=2000$ and shows the conditions of their appearance as a function of $h$. Note that for the $R a$ numbers considered, the fluid along the hot cylinder moves upward to reach the fin and tends to jump it, but it is guided along the fin until meeting the cold wall and then descends along the outer cylinder creating a nearly stagnant flow area in the upper space especially for low and intermediate heights. The intensity of the secondary cell does not exceed $3.2 \%$ of the intensity of the main cell at $h=0.515$ for $R a=2790$. For higher $R a(2800 \leq R a \leq 3770)$, it is worth noting that the appearance of the BCR regime is intermittent.
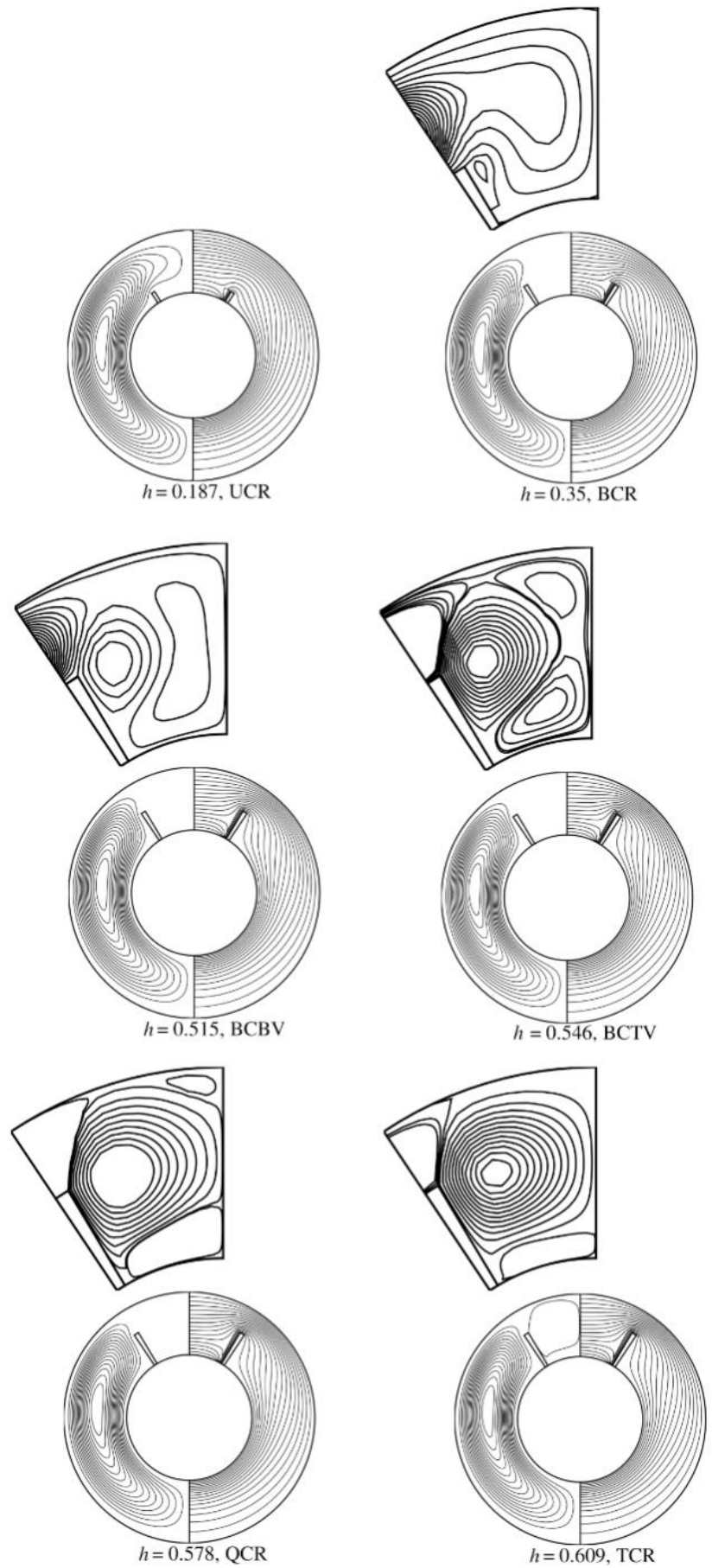

(a) 

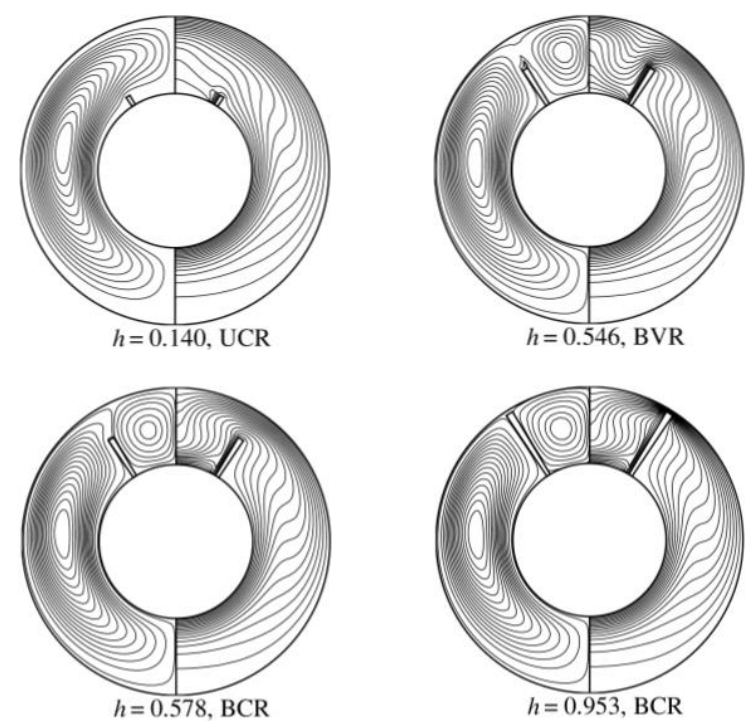

(b)

Figure 2. (a) Streamlines and isotherms for $R a=2000$; (b) Streamlines and isotherms for $R a=7000$

A bi-vortex regime BVR interposes for restraint height intervals: $0.328 \leq h \leq 0.453$ for $R a=3000$ and $0.296 \leq h \leq$ 0.453 for $R a=3500$ and at $0.281 \leq h \leq 0.453$ for $R a=3770$.

For $R a>3770$, the increase in $h$ leads to the appearance of two regimes: bi-vortex BVR, then bicellular BCR. Figure 2 (b) shows the appearance of these two structures for $R a=7000$. Summarizing results of similar flow regimes for $3770<R a \leq$ 10000 , Figure 3 presents in the $h-R a$ plane the transition lines between all flow structures described above.

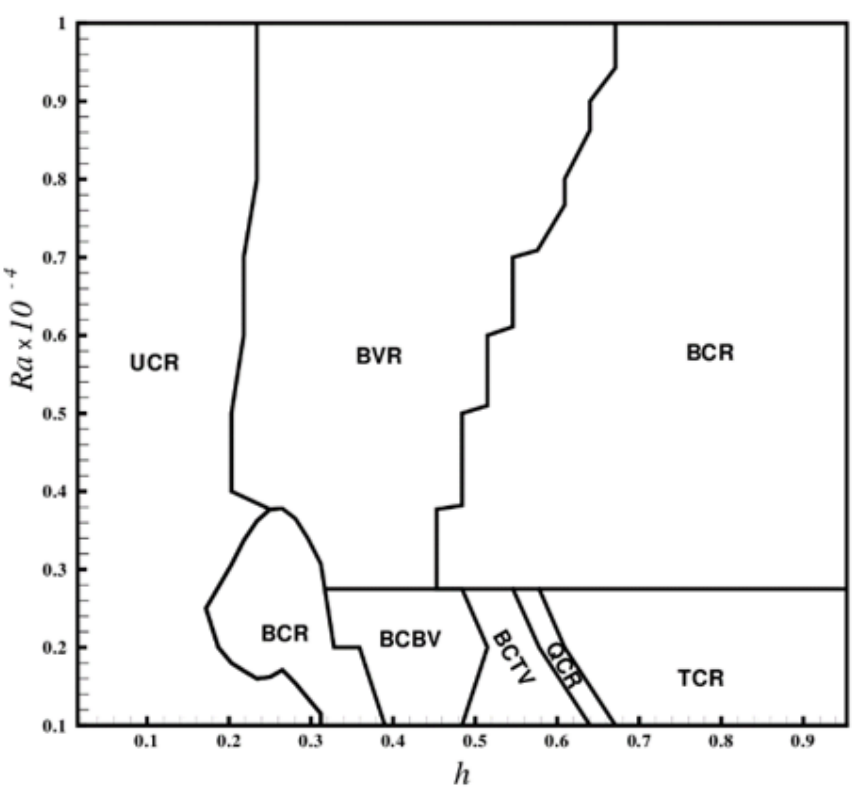

Figure 3. Flow regimes in the $h-R a$ plane

The Nusselt number variation curves, $\overline{N u}$, as a function of $h$, shown in Figure 4 (a), indicate that for each $R a, \overline{N u}$ decreases with increasing $h$ and reaches a minimum. The maximum of decrease is noted for $R a=10000$, in the order of $5.32 \%$. For $1000 \leq R a \leq 2790, \overline{N u}$ increases with $h$ independently of the different flow regimes that are established. Except for $R a \geq$ 2670 where the flow regimes start to influence the $\overline{N u}$ curves. For $2800 \leq R a \leq 3770 \overline{\mathrm{Nu}}$ undergoes a jump in the transition to the BCR regime, equal to $8.16 \%$ at $h=0.203$ for $R a=3000$ and $13.06 \%$ at $h=0.265$ for $R a=3770$, then decreases rapidly at the transition BCR-BVR. This jump in the curve of $\overline{N u}$ as a function of $h$ corresponds respectively to the UCR-BCR regime change for the increase of $\overline{N u}$ then to the BCR-BVR for the decrease (Figure 3). Afterwards $\overline{N u}$ increases gradually, undergoes an accentuated rise related to the BVR-BCR transition, and reaches its maximum at $h=0.953$. For heights, $0.062 \leq h$ for $R a=10^{3}$ and $0.390 \leq h$ for $R a=10^{4}$, the decrease of $\overline{N u}$ can be related to the blocking effect of the flow by the fins that outweighs their heating effect, which can be explained by the fact that the heat exchange surface has not increased much. For the relatively higher heights $\overline{N u}$ increases as a function of $h$ indicating that the effect of heating by fins is dominant. The increase of $\overline{N u}$ when $h$ goes from 0.25 to 0.5 and then to 0.75 is in agreement with the finding of Farinas et al. [9].

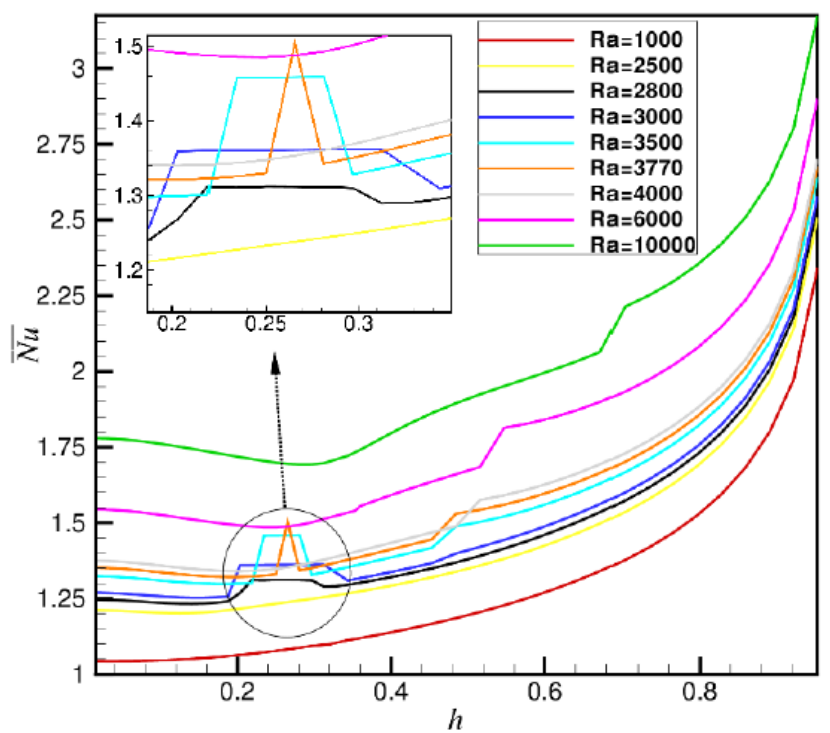

(a)

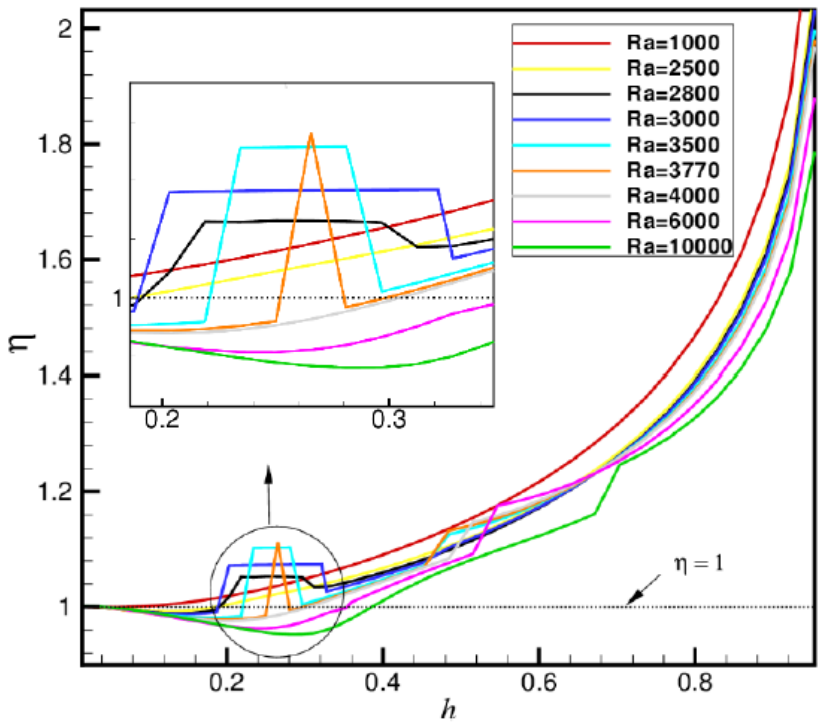

(b)

Figure 4. (a) Variation of the average Nusselt number as a function of $h$; (b) Variation of the effectiveness as a function of $h$

The effectiveness of the system, $\eta$, is defined as the ratio of the average Nusselt number for the configuration with fins to the average Nusselt number for the configuration without fins 
$\eta=\frac{\overline{N u} \text { with fins }}{\overline{N u} \text { without fins }}$. Figure 4 (b) shows the variation of $\eta$ as a function of $h$ and shows that for the very low heights it is almost equal to 1 . Afterwards it decreases with the increase of $h$, then increases to the limit value of 1 indicating that the configuration without fins can be considered as being favorable to the heat transfer rate for intervals of $h$ obtained as a function of $R a$, from $h=0.0$ to $h=0.062$ for $R a=10^{3}$ and to $h=0.390$ for $R a=10^{4}$. As $h$ increases, $\eta$ rises and undergoes two jumps corresponding to the two transitions towards the BCR regime. From $h=0.390, \eta$ becomes strictly greater than 1 for all $R a$ indicating that the heat transfer rate is greater in these cases relatively to the finless case. Farinas et al. [9] showed that the effectiveness increases with the increase of the fin height, and in some cases $\eta$ is slightly less than 1 in the sixfin configuration with $h=0.25$. Whereas in the present study the increment of $h$ is much smaller, 0.015 , for heights ranging from 0.015 to 0.953 , which allowed the observation of the decrease of $\eta$ as a function of $h$ in the cases cited above.

In addition, for $2800 \leq R a \leq 3770$ the fin heights taken in intervals: $0.218 \leq h \leq 0.296$ for $R a=2800,0.203 \leq h \leq 0.312$ for $R a=3000$ and $0.234 \leq h \leq 0.281$ for $R a=3500$ as well as $h=0.265$ for $R a=3770$, offer a better effectiveness than that obtained for all the other values of $R a$, in the same intervals of $h$, for cases with and without fins. This finding is certainly related to the presence of the BCR regime already mentioned in these intervals. For the other Rayleigh numbers, Figure 4 (b) shows that for $R a=10^{3}$ the effectiveness is greater than that obtained for the heights considered. In their studies with other configurations, Farinas et al. [9, 10] and Elatar et al. [13] found that it is at $R a=10^{3}$ where the effectiveness is greatest compared to that calculated at the other higher $R a$. These authors justify their results referring to the conductive heat transfer, which is dominant for $R a=10^{3}$ and the fin blockage effect which is negligible. Note that in the present study, similar conclusions would have been drawn if only three particular values of $h$ had been selected, for example: $h=0.25$, 0.5 and 0.75 (see Figure 4 (b)), but acting with the very small increment of $h$ equal to 0.015 allows to find out more precise results, and subsequently provides the possibility to increase or decrease the heat transfer in the cavity.

Regarding the effect of $R a$ on the average Nusselt number for the different heights studied, Figure 5 (a) shows that $\overline{N u}$ generally increases with $R a$. The establishment of the different flow regimes does not indicate a remarkable change in the variation curve of $\overline{N u}$ as a function of $R a$ except for $0.203 \leq h$ $\leq 0.312$ where an increase of $\overline{N u}$ is noticed, related to the BCR regime, when the secondary cell grows rapidly for a slight variation of $R a$. At the disappearance of the BCR regime for $0.203 \leq h \leq 0.312$ and $0.484 \leq h \leq 0.671, \overline{N u}$ undergoes a noteworthy fall. It can be concluded that the increase of the secondary cell and the disappearance of the BCR regime have a significant influence on the variation of $\overline{N u}$. Note that for the finless case $\overline{N u}$ increases also with the rise of $R a$, and remains lower than that obtained for the height limit $h=0.390 . \overline{N u}$ for this height is equal to 1.1333 at $R a=1000$ and 1.7831 at $R a=$ 10000. For the finless case, $\overline{N u}$ is 1.0436 at $R a=1000$ and 1.7766 at $R a=10000$.

For heights $h<0.390$, the heat transfer rate may be lower than that of the case without fins, depending on the value of $R a$, Figure 5 (b). The configuration without fins is in this case advantageous to heat transfer rate. When $h \geq 0.390$ the fin configuration remains more favorable to heat transfer rate regardless of the Rayleigh number, despite the fall in heat transfer rate at the disappearance of the BCR regime for heights $0.484 \leq h \leq 0.671$. The effectiveness for $h=0.390$ compared to the finless case remains greater than 1 when the Rayleigh number varies from 1000 to $10000 . \eta$ is equal to 1.004 at $R a=10000$.

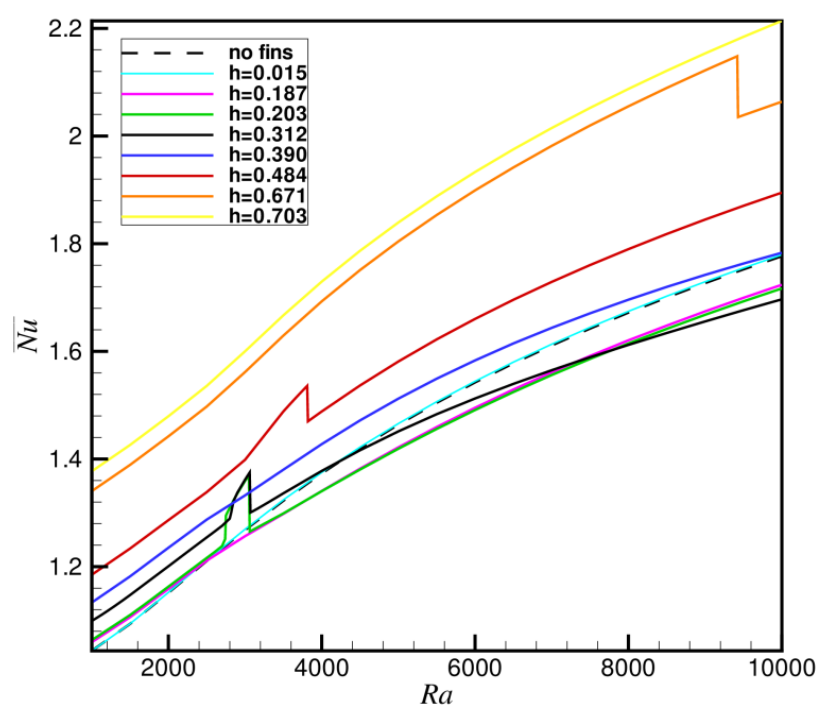

(a)

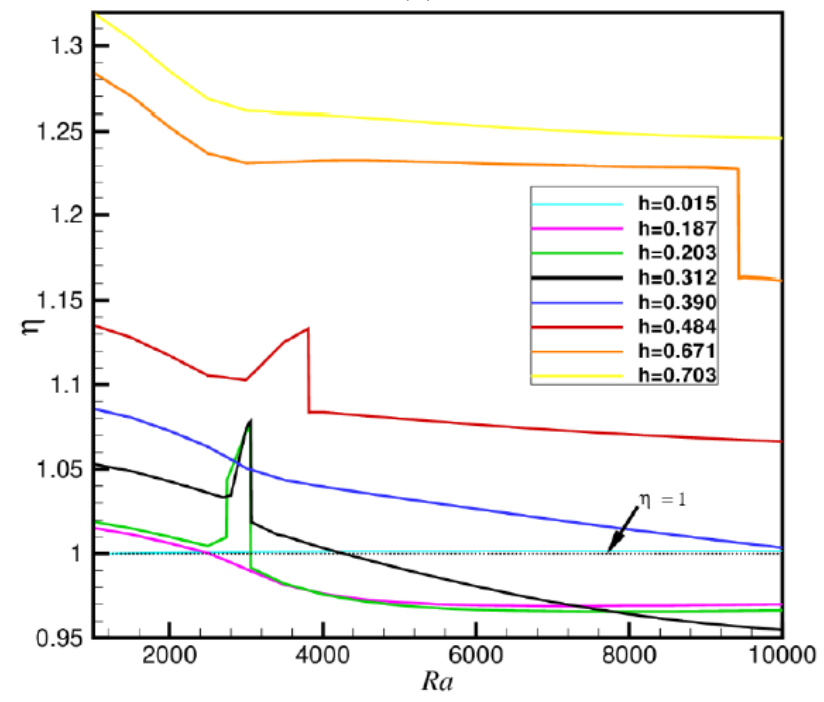

(b)

Figure 5. (a) Variation of the average Nusselt number as a function of $R a$ for $0.015 \leq h \leq 0.953$; (b) Variation of the effectiveness as a function of $R a$ for $0.015 \leq h \leq 0.953$

Figure 6 (a) summarizes the results of the average Nusselt number and the effectiveness in the $h-R a$ plane through the abacus giving the iso-value lines of $\overline{N u}$ and the effectiveness divided into three zones:

Zone I: The increase in the height of the fins for a given Rayleigh number leads to a decrease in the Nusselt number with a minimum observed at the heights identified by the line L1. Then $\overline{N u}$ increases and reaches the height limit indicated by the line $L 2\left(0.062\right.$ for $R a=10^{3}$ and 0.390 for $\left.R a=10^{4}\right)$ for which it is equivalent to that obtained for $h=0.015$. As an example, for $R a=5000, \overline{N u}$ decreases from 1.47 to 1.42 between $h=0.015$ and $h=0.218$, then increases to 1.47 for $h$ $=0.328$ (point A), a value identical to that obtained for $h=$ 0.015 . As a result, in this zone limited by $L 2$, despite the UCRBVR transition, the height $h=0.015$ is more favorable to the 
heat transfer rate compared to higher heights but remains less favorable than or as favorable as the values marked by $L 2$. Since the effectiveness for this height is almost equal to 1 (Figure 6 (b)), the case without fins is recommended because of its simpler design. The use of fins in Zone I is recommended only if the objective is to reduce heat transfer rate.

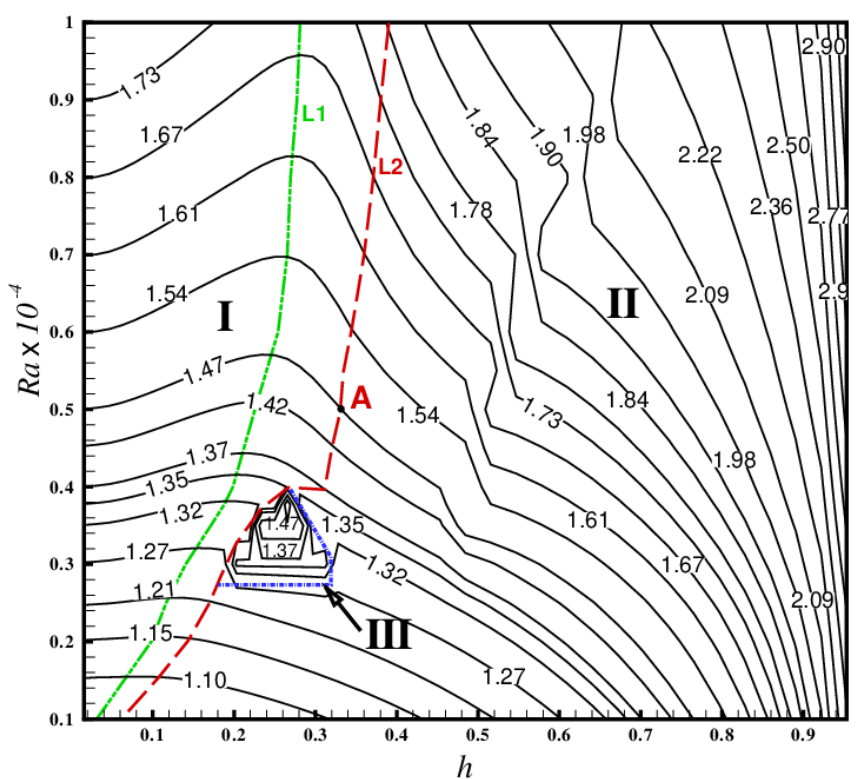

(a)

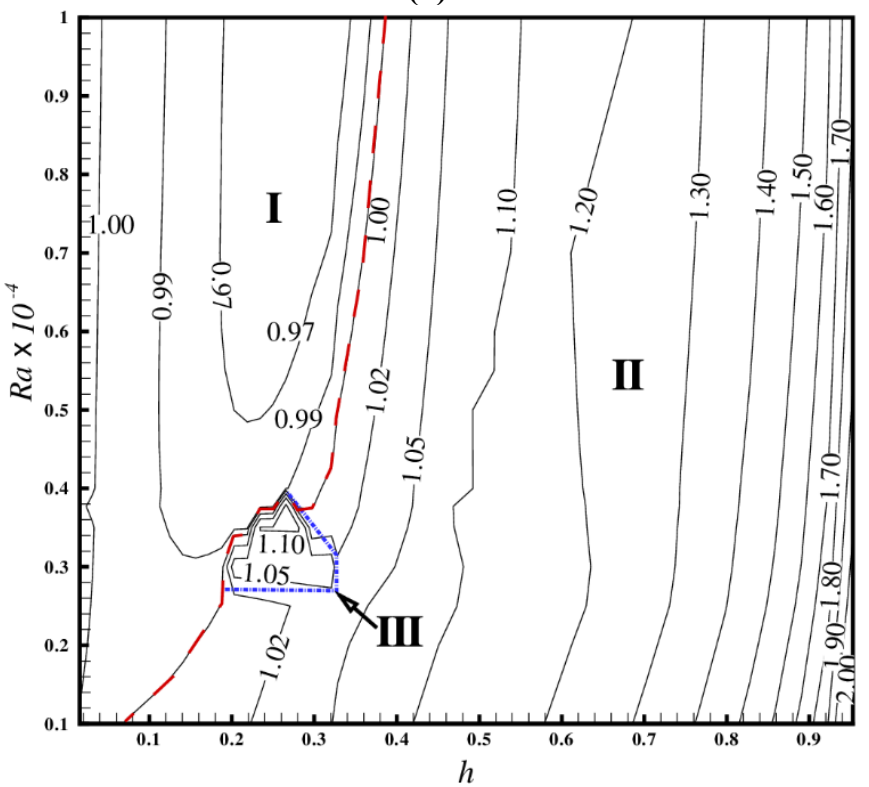

(b)

Figure 6. (a) Average Nusselt number in the $h$-Ra plane; (b) Effectiveness in the $h-R a$ plane

Zone II: This zone limited by heights strictly greater than the values indicated by $L 2$, shows that for a constant Rayleigh number, $\overline{\mathrm{Nu}}$ increases with the increase of $h$. A significant increase in $\overline{N u}$ is noticed at the BVR-BCR transition. The other transitions between the different regimes observed for weak values of $R a$ do not correspond to a remarkable change of $\overline{N u}$. In this case, the increase in height increases the heat transfer rate regardless of $R a$. The same observation is made on the enhancement of the effectiveness as a function of $h$ in this zone, Figure 6 (b).

Zone III: This particular zone in the form of a triangle where the flow regime is $\mathrm{BCR}$ indicates that for a given $R a$ number,
$\overline{N u}$ remains almost constant. As an example, at $R a=3000, \overline{N u}$ is equal to 1.35 for heights ranging from $h=0.203$ to $h=0.312$. In addition, the effectiveness is greater than 1 and to that obtained at $R a=1000$ and at Rayleigh numbers greater than 3770 .

On the other hand, for a constant value of $h, \overline{N u}$ has an identical value for two distinct Rayleigh numbers. At $h=0.234$ $\overline{N u}$ is equal to 1.47 at $R a=3500$ and at $R a=5500$.

\section{CONCLUSIONS}

Laminar natural convection inside an air-filled cylindrical annular space of radius ratio $R=2$, fitted with two thin fins attached to the hot wall at the position $\varphi_{m}=0.82 \pi$ has been studied numerically. The height of the fins $h$ varies from 0.015 to 0.953 and Rayleigh number $R a$ is ranging from $10^{3}$ to $10^{4}$. The present study focuses on the parameters $h$ and $R a$, that affect heat transfer. The numerical results obtained show that increasing $h$ can favor or disadvantage the heat transfer. It also shows that increasing $R a$, is not necessarily synonymous with increased heat transfer rate nor with decreased effectiveness. These results could be obtained due to the choice of very small increments equal to 0.015 for $h$ and 10 for $R a$, which gives more reliable results than those obtained with larger increments studied by other authors. The following conclusions can be deduced from this study:

1. For heights below a limit value according to $R a$ ( 0.062 for $R a=10^{3}$ and 0.390 for $R a=10^{4}$ ), the height $h=$ 0.015 gives a maximum average Nusselt number that is almost identical to that obtained for $h=0.0$. The absence of fins gives therefore the greatest heat transfer and consequently the effectiveness is less than 1 . Beyond these limit values, Nusselt number grows with $h$, the configuration with fins becomes more and more favorable to heat transfer.

2. For heights in a small range around $h=0.25$ and for $2800 \leq R a \leq 3770$, the effectiveness is greater than 1 and to that obtained for the other values of $R a$.

3. For heights $0.203 \leq h \leq 0.312$ and $0.484 \leq h \leq 0.671$, the increase of Rayleigh number with a tight increment leads to the disappearance of the BCR regime, with a subsequent decrease in heat transfer rate.

\section{REFERENCES}

[1] Shen, Q., Sun, D., Xu, Y., Jin, T., Zhao, X., Zhang, N., Wu, K., Huang, Z. (2016). Natural convection heat transfer along vertical cylinder heat sinks with longitudinal fins. International Journal of Thermal Sciences, 100: 457-464. https://doi.org/10.1016/j.ijtherm alsci.2015.09.007

[2] Chai, J.C., Patankar, S.V. (1993). Laminar natural convection in internally finned horizontal annuli. Numerical Heat Transfer, Part A: Applications, 24(1): 67-87. https://doi.org/10.1080/10407789308902603

[3] Rahnama, M., Mehrabian, M.A., Mansouri, S. H., Sinaie, A., Jafargholi, K. (1999). Numerical simulation of laminar natural convection in horizontal annuli with radial fins. Proceedings of the Institution of Mechanical Engineers, Part E: Journal of Process Mechanical Engineering, 213(2): 93-97. https://doi.org/10.1243/0954408991529780

[4] Rahnama, M., Farhadi, M. (2004). Effect of radial fins 
on two-dimensional turbulent natural convection in a horizontal annulus. International Journal of Thermal Sciences, 43(3): 255-264. https://doi.org/10.1016/j.ijther malsci.2003.07.002

[5] Jafari, I., Mahdavy-Moghadam, H., Taeibi-Rahni, M., Dayyan, M., Seyyedi, S.M. (2014). Effect of radial fins on natural convection between horizontal circular and square cylinders. Journal of Theoretical and Applied Mechanics, 52(3): 827-837.

[6] Rustum, I.M., Soliman, H.M. (1990). Numerical analysis of laminar mixed convection in horizontal internally finned tubes. International Journal of Heat and Mass Transfer, 33(7): https://doi.org/10.1016/0017-9310(90)90045-V

[7] Mirza, S., Soliman, H.M. (1985). The influence of internal fins on mixed convection inside horizontal tubes. International Communications in Heat and Mass Transfer, 12(2): 191-200. https://doi.org/10.1016/07351933(85)90067-3

[8] Abu-Hijleh, B.A.K. (2003). Optimized use of baffles for reduced natural convection heat transfer from a horizontal cylinder. International Journal of Thermal Sciences, $\quad 42(11)$ : 1061-1071. https://doi.org/10.1016/S1290-0729(03)00089-9

[9] Farinas, M.I., Garon, A., St-Louis, K. (1997). Study of heat transfer in a horizontal cylinder with fins. Revue Générale de Thermique, 36(5): 398-410. https://doi.org/10.1016/S0035-3159(97)81601-7

[10] Farinas, M.I., Garon, A., St-Louis, K., Lacroix, M. (1999). Study of heat transfer in horizontal bare and finned annuli. International Journal of Heat and Mass Transfer, $\quad$ 42(21): 3905-3917. https://doi.org/10.1016/S0017-9310(99)00077-0

[11] Alshahrani, D., Zeitoun, O. (2005). Natural convection in horizontal cylindrical annuli with fins. Alexandria Engineering Journal, 44(6): 825-837.

[12] Zeitoun, O., Hegazy, A.S. (2004). Heat transfer for laminar flow in internally finned pipes with different fin heights and uniform wall temperature. Heat and Mass Transfer, 40: 253-259. https://doi.org/0.1007/s00231003-0446-8

[13] Elatar, A., Teamah, M.A., Hassab, M.A. (2016). Numerical study of laminar natural convection inside square enclosure with single horizontal fin. International Journal of Thermal Sciences, 99: 41-51. https://doi.org/10.1016/j.ijthermalsci.2015.08.003

[14] Sheikhzadeh, G.A., Arbaban, M., Mehrabian, M.A. (2013). Laminar natural convection of $\mathrm{Cu}$-water nanofluid in concentric annuli with radial fins attached to the inner cylinder. Heat Mass Transfer, 49: 391-403. https://doi.org/10.1007/s00231-012-1084-9

[15] Sultan, K.F. (2015). Numerical solution of heat transfer and flow of nanofluids in annulus with fins attached on the inner cylinder. Journal of Babylon University/Engineering Sciences, 23(2): 465-484.

[16] Kiwan, S., Zeitoun, O. (2008). Natural convection in a horizontal cylindrical annulus using porous fins. International Journal of Numerical Methods for Heat \& Fluid, $\quad$ 18(5): 618-634 https://doi.org/10.1108/09615530810879747

[17] Masliyah, J.H., Nandakumar, K. (1976). Heat transfer in internally finned tubes. Journal of Heat Transfer, 98(2): 257-261. https://doi.org/10.1115/1.3450528

[18] Syed, K.S., Iqbal, Z., Ishaq, M. (2011). Optimal configuration of finned annulus in a double pipe with fully developed laminar flow. Applied Thermal Engineering, $\quad 31(8-9)$ : $1435-1446$. https://doi.org/10.1016/j.applthermaleng.2011.01.012

[19] Iqbal, Z., Syed, K.S., Ishaq, M. (2015). Optimum convection through parabolic fins in annulus of double pipe. Journal of Thermophysics and Heat Transfer, 29(3): 572-586. https://doi.org/10.2514/1.T4420

[20] Iqbal, Z., Syed, K.S., Ishaq, M. (2017). Optimum configurations of annulus with triangular fins for laminar convection. Thermal Science, 21(1A): 161-173. https://doi.org/10.2298/TSCI130805139

[21] Idrissi, A., Cheddadi, A., Ouazzani, M.T. (2016). Heat transfer in an annular space fitted with heating isothermal blocks: Numerical bifurcation for low blocks height. Case Studies in Thermal Engineering, 7: 1-7. https://doi.org/10.1016/j.csite.2015.11.002

[22] Cheddadi, A., Caltagirone, J.P., Mojtabi, A., Vafai, K. (1992). Free two-dimensional convective bifurcation in a horizontal annulus. Journal of Heat Transfer, 114(1): 99106. https://doi.org/10.1115/1.2911274

[23] Taher, Y., Cheddadi, A., Ouazzani, M.T. (2011). Natural convection flow regimes in an annular space provided with fins-Transition diagram. Physical and Chemical News, 57: 76-80.

\section{NOMENCLATURE}$$
g
$$

$h$

$\overline{N u}$

$\operatorname{Pr}$

$R a$

$R$

$r$

$r_{o}$

$r_{i}$

$T_{o}$

$T_{i}$

$t$

$U, V$

$w$

gravitational acceleration, $\mathrm{ms}^{-2}$

dimensionless height of the fins

average Nusselt number

Prandtl number

Rayleigh number

radii ratio

dimensionless radial coordinate

outer cylinder radius, $m$

inner cylinder radius, $m$

outer cylinder temperature, $\mathrm{K}$

inner cylinder temperature, $\mathrm{K}$

dimensionless time

dimensionless velocity components respectively in the directions $r$ and $\varphi$ dimensionless thickness of the fins

\section{Greek symbols}

$\alpha$

$\beta$

$\eta$

$\nu$

$\varphi$

$\varphi_{m}$

$\psi$

$\omega$

\section{Subscripts}

$i \quad$ inner thermal diffusivity, $\mathrm{m}^{2} \mathrm{~s}^{-1}$ thermal expansion coefficient, $\mathrm{K}^{-1}$ effectiveness of the system kinematic viscosity, $\mathrm{m}^{2} \mathrm{~s}^{-1}$ polar angle angular position of the fins dimensionless stream function dimensionless vorticity 